\title{
Knowledge, attitudes and practices on malaria transmission in Mamfene, KwaZulu- Natal Province, South Africa 2015
}

\author{
Pinky N. Manana ${ }^{1,2^{*}}$, Lazarus Kuonza ${ }^{1,2}$, Alfred Musekiwa ${ }^{1,3}$, Hluphi D. Mpangane ${ }^{1}$ and Lizette L. Koekemoer ${ }^{4,5}$
}

\begin{abstract}
Background: In South Africa malaria is endemic in Mpumalanga, Limpopo and the north-eastern areas of KwaZulu-Natal provinces. South Africa has set targets to eliminate malaria by 2018 and research into complementary vector control tools such as the Sterile Insect Technique (SIT) is ongoing. It is important to understand community perceptions regarding malaria transmission and control interventions to enable development of community awareness campaign messages appropriate to the needs of the community. We aimed to assess knowledge, attitudes, and practices regarding malaria transmission to inform a public awareness campaign for SIT in Jozini Local Municipality, Mamfene in KwaZulu-Natal province.
\end{abstract}

Methods: We conducted a cross-sectional survey in three communities in Mamfene, KwaZulu-Natal during 2015. A structured field piloted questionnaire was administered to 400 randomly selected heads of households. Descriptive statistics were used to summarize data.

Results: Of the 400 participants interviewed, $99 \%$ had heard about malaria and correctly associated it with mosquito bites. The sources of malaria information were the local health facility (53\%), radio (16\%) and community meetings (7\%). Approximately $63 \%$ of the participants were able to identify three or four symptoms of malaria. The majority (76\%) were confident that indoor residual spraying (IRS) kills mosquitoes and prevents infection. Bed nets were used by $2 \%$ of the participants. SIT knowledge was poor (9\%), however $63 \%$ of the participants were supportive of mosquito releases for research purposes. The remaining $37 \%$ raised concerns and fears, including fear of the unknown and lack of information on the SIT.

Conclusion: Appropriate knowledge, positive attitude and acceptable treatment-seeking behaviour for malaria were demonstrated by members of the community. Community involvement will be crucial in achieving success of the SIT and future studies should further investigate concerns raised by the community. The existing communication channels used by the malaria control program can be used; however additional channels should be investigated.

Keywords: Malaria transmission, Knowledge, Attitude and practices, Sterile-insect technique, Survey, South Africa, KwaZulu-Natal

\footnotetext{
* Correspondence: pinkym@nicd.ac.za

'South African Field Epidemiology Training Programme, National Institute for

Communicable Diseases, Johannesburg, South Africa

${ }^{2}$ School of Health Systems and Public Health, Faculty of Health Sciences,

University of Pretoria, Johannesburg, South Africa

Full list of author information is available at the end of the article
} 


\section{Background}

Malaria is a public health challenge with approximately 214 million cases and 438,000 deaths occurring globally in 2015 [1, 2]. Africa accounts for over $80 \%$ of cases and $90 \%$ of deaths in the world with sub-Saharan Africa being the most affected malaria region $[1,2]$. In South Africa (SA) the incidence of malaria was estimated at 20 cases per 100,000 population in 2015 [3]. The majority of the reported malaria cases in SA are currently attributed to Plasmodium falciparum infections, predominantly transmitted by Anopheles arabiensis, although $A n$. funestus was implicated in the past [4]. The Malaria Control Programme (MCP) in SA focuses on vector control and case management $[4,5]$.

Vector control is primarily based on indoor residual spraying (IRS) [6, 7]. The IRS technique has been in operation effectively since the 1940s; however it has failed to completely eliminate malaria [7]. Limitations to the technique have been partly due to its limited suitability for indoor treatment and to insecticide resistance [8]. Insecticides that are currently used for IRS in SA include dicloro-diphenyl-trichloroethane (DDT) and the pyrethroid deltamethrin [4, 7]. South Africa is currently targeting elimination of malaria $[1,5]$. However, additional strategies are needed to strengthen the current IRS based vector control intervention to achieve elimination. One such strategy is the Sterile Insect Technique (SIT) [9].

The SIT is an environmentally friendly, area-wide integrated pest management method [10]. Research conducted since the mid-1950s has shown SIT as an effective strategy in suppressing targeted insect populations, including mosquitoes [9]. The SIT process involves the inundative release of sterile males at high enough rates to cause a decline in a target wild vector population. It has been used successfully in the control of a number of insect pest species across the world: control of the Mediterranean fruit fly, Ceratitis capitata [11], eradication of tsetse fly Glossina austeni in the Island of Zanzibar [12] and eradication of the New World screwworm fly Cochliomyia hominivorax in the USA and Mexico [13, 14]. South Africa has been successfully using SIT to control the Mediterranean fruit fly in the Hex River Valley in the Western Cape since 1999 [15]. The feasibility of SIT as a malaria intervention tool is under investigation in several countries, including SA [16].

Prior studies conducted on malaria vector that circulate in the northern region of KwaZulu-Natal (KZN) province have reported increasing resistance to insecticides that are used for IRS $[17,18]$. These findings have highlighted the need to implement additional/alternative vector control methods in order to sustain the reduction in malaria transmission in the population. Accordingly, the Mamfene area of northern KZN has been earmarked for a pilot study to assess the feasibility of using SIT for malaria vector control.

However, before a pilot programme is implemented, it is important to increase awareness about the programme in the target communities to ensure adequate cooperation and participation. Previous studies have demonstrated that directly engaging the community plays an important role in improving the acceptability and effectiveness of programmes aimed at reducing the transmission of malaria [9, 19]. Failure to consider the beliefs and perceptions of the community regarding aspects of the planned programmes may lead to negative attitudes or practices and contribute to failure to achieve the intended goals [9]. The SIT trials in the 1970s in New Delhi, India, to eradicate Culex quinquefasciatus, failed due to negative publicity in the community [9] and another trial in El Salvador, to eradicate Anopheles albimanus, was disrupted due to civil unrest [20]. Understanding community awareness of malaria transmission and concerns regarding control activities will allow research investigations to be modified to suit the needs of the community.

In order to provide community level baseline information and to inform public awareness campaigns specifically aimed at SIT we aimed to assess the knowledge, attitudes and practices of the Mamfene community in $\mathrm{KZN}$ regarding the transmission, prevention and treatment of malaria.

\section{Methods}

\section{Study design and setting}

We conducted a descriptive cross-sectional household survey during October 2015, in the Mamfene area of Jozini Local Municipality, which is situated in uMkhanyakude district of KwaZulu-Natal Province (Fig. 1). The district has five local municipalities including uMhlabuyalingana, Jozini, the Big Five False Bay, Hlabisa and Mtubatuba [21]. In 2011, the population in Jozini Local Municipality was estimated at 186,502 (29.8\%) with $\sim 39,000$ households (in comparison to the other municipalities) [21] made up of the following age groups: 014 years (41.3\%), 15-64 years $(54.8 \%)$ and $>65$ years (3.9\%). Over half $(54 \%)$ of the population were females [22]. Mamfene comprises of 10 sections where Indoor Residual Spraying (IRS) is conducted annually and when a need has been identified. This study concentrated on three of the ten sections in Mamfene, Sections 2, 8 and 9. The three sections already serve as sentinel entomological surveillance sites for the KZN malaria control programme (MCP), and all three have also been selected for the assessment of the feasibility of implementing SIT. The population in the three sections was: section 2 $(n=2024)$; section $8(n=4592)$ and section 9 ( $n=2167)$ [21-23]. 


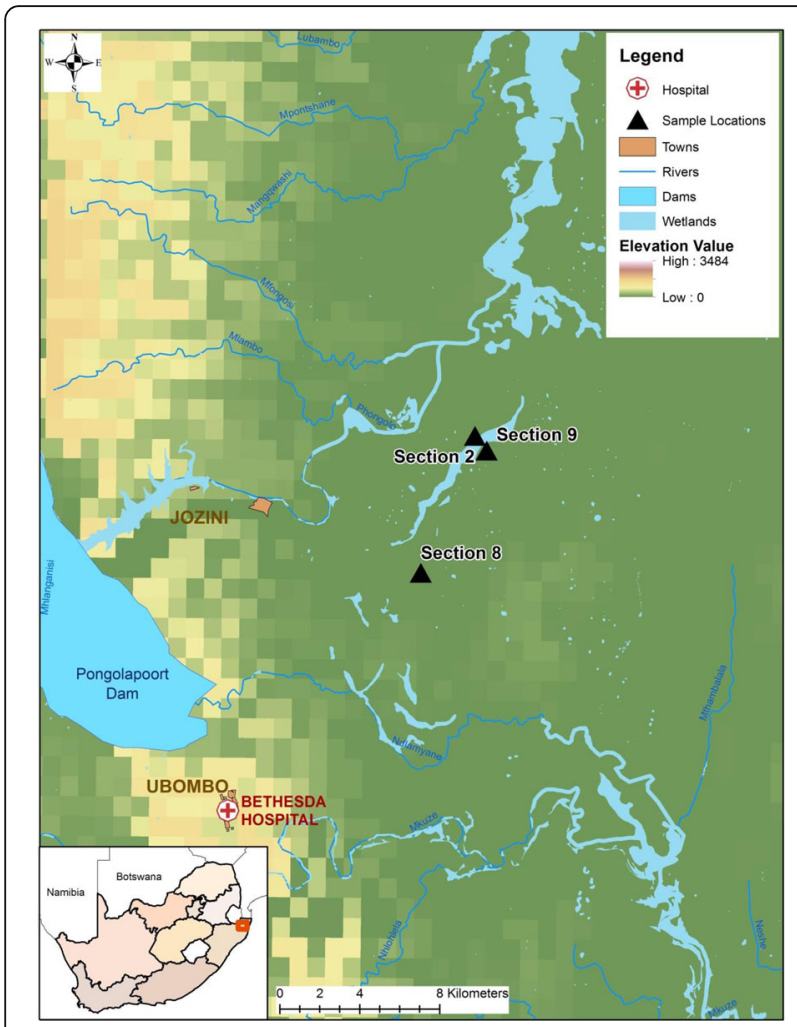

Fig. 1 Map of Jozini Local Municipality showing the three sections in Mamfene, KwaZulu-Natal, South Africa (map adapted from Dandalo et al.: unpublished data)

\section{Data collection}

Trained field workers administered a pre-piloted questionnaire that was translated into IsiZulu, the local language of the area, to respondents aged 18 years and older. The questionnaire was adapted from a tool that was previously used during a community KAP study on malaria in Swaziland in 2007 [24]. The final survey questionnaire was divided into five sections: demographics of the respondent; knowledge; attitudes; social practices and treatment-seeking behaviour related to malaria transmission and disease; and SIT. The questionnaire included closed-ended, partially closed and open-ended questions (the study questionnaire is available in supplementary file 1). The questionnaire was administered to 400 adult respondents. One key respondent, 18 years and older, was identified for each randomly selected household and preference was given to the head of the household when available.

\section{Sample size and sampling plan}

The total population of the three sections was $N=8783$. Since the proportions of knowledge, attitudes and practices regarding malaria transmission in this setting was unknown, a $50 \%$ proportion was assumed to ensure the maximum sample size. A sample size of $n=384$ was calculated using Epi Info ${ }^{\mathrm{Tm}}$ 7.1.4.0 assuming a population size of $N=9000,50 \%$ prevalence, $95 \%$ confidence interval and $5 \%$ precision. We assumed a $96 \%$ response that resulted in a final sample size of 400 households.

The total number of households was allocated to the three sections proportional to population size, giving sample sizes of $n=90, n=210$ and $n=100$ for sections 2, 8 and 9, respectively. Each section consists of six sentinel sites; all of which were used in the study. The sample size for each section was divided among the six sentinel sites such that each sentinel site contributed proportionate participants (15 study participants per site in section 2, 35 participants per site in section 8 and 17 participants per site in section 9).

\section{Data analysis}

Data were captured on Epi Info ${ }^{\mathrm{mm}}$ 7.1.4.0 and exported to Excel. Descriptive statistical analysis was carried out using STATA Version 13. Measures of central tendency and dispersion were calculated for quantitative variables, and proportions were calculated for categorical variables. Frequency distribution tables and appropriate charts were displayed to show differences in the relative frequencies of variables. A Chi square test was used to determine whether there was a significant relationship between categorical variables. A $p$ value $<0.05$ was considered statistically significant.

\section{Results}

A total of 400 questionnaires were completed, with no refusals and $100 \%$ of questions completed. The majority of the participants were females $71 \%(n=285)$. Ages of the participants ranged between 18 and 90 years old, with a mean age $(\mathrm{SD})$ of $42( \pm 16.5)$ years. Heads of household accounted for $37 \%(n=149)$ of the respondents. The majority of the respondents, $71 \%(n=286)$ were unemployed and most had attained at least primary level education, $71 \%(n=284)$. In addition, $12 \%(n=47)$ of the respondents reported that at least one family member had parasitological confirmed (by Rapid Diagnostic Test) malaria in the preceding year (2014) (Table 1).

\section{Knowledge of malaria transmission}

Of the 400 participants interviewed, 99\% (95\% CI: 97.899.8\%) had heard about malaria before and correctly associated malaria with mosquito bites. All participants who had heard about malaria further reported that malaria can kill if it is not treated. Only 32\% (95\% CI: 27.4$36.8 \%$ ) associated the female mosquito as the carrier of infection. The most commonly identified source of malaria information were the local health facility 53\% (95\% CI: $48.2-58.2 \%$ ), radio $15 \%$ (95\% CI: $12.0-19.4 \%)$ and community meetings $7 \%$ (95\% CI: 4.9-10.2\%), with community health promoters and pamphlet distribution 
Table 1 Demographic characteristics and malaria history among participants in Mamfene, Jozini, KwaZulu-Natal, South Africa 2015

\begin{tabular}{|c|c|c|}
\hline Characteristics & $n=400$ & Percent \\
\hline \multicolumn{3}{|l|}{ Gender } \\
\hline Male & 115 & 28.8 \\
\hline Female & 285 & 71.2 \\
\hline \multicolumn{3}{|l|}{ Age (in years) } \\
\hline $18-35$ & 163 & 40.8 \\
\hline $36-50$ & 111 & 27.7 \\
\hline $51-65$ & 78 & 19.5 \\
\hline$>65$ & 45 & 12.0 \\
\hline \multicolumn{3}{|l|}{ Family position } \\
\hline Head of household & 149 & 37.2 \\
\hline Wife & 112 & 28.0 \\
\hline Children & 129 & 32.3 \\
\hline Other & 10 & 2.5 \\
\hline \multicolumn{3}{|c|}{ Highest level of education attained } \\
\hline No schooling & 116 & 29.0 \\
\hline Primary level & 131 & 32.7 \\
\hline Secondary level & 144 & 36.1 \\
\hline Tertiary level & 9 & 2.2 \\
\hline \multicolumn{3}{|l|}{ Employment status } \\
\hline Employed & 21 & 5.3 \\
\hline Unemployed & 286 & 71.5 \\
\hline Housewife & 15 & 3.8 \\
\hline Pensioner & 71 & 17.7 \\
\hline Seasonal worker & 1 & 0.2 \\
\hline Student & 6 & 1.5 \\
\hline \multicolumn{3}{|c|}{ Family history of malaria in $2014^{a}$} \\
\hline Had malaria infection & 47 & 11.8 \\
\hline No malaria infection & 352 & 88.0 \\
\hline Do not know & 1 & 0.2 \\
\hline
\end{tabular}

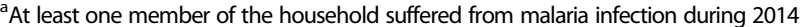

contributing only less than $1 \%$ (Fig. 2). Approximately 59\% (95\% CI: 54.5-64.3\%) reported not to have enough information on malaria and were keen to know more.

The most frequently reported signs and symptoms of malaria included headaches (78\%), fever (54\%), feeling cold (60\%) and vomiting (30\%). Approximately $63 \%$ of the participants were able to correctly identify three or four of the symptoms (Fig. 3). Only $9 \%(n=36)$ of the participants had heard about the SIT. However, the majority of the participants, $63 \%$ (95\% CI: $57.8-67.5 \%$ ), had a positive response with regards to releasing mosquitoes into the environment for research purposes, after the study team described the purpose and practice of SIT to them (Table 2).

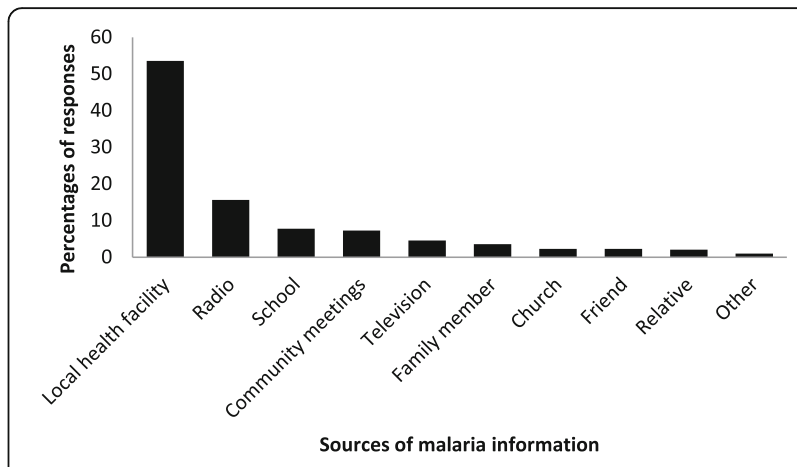

Fig. 2 Sources of malaria information as identified by the participants in Mamfene Jozini, KwaZulu-Natal, South Africa, 2015

The 18-50 years age group were more in support of the SIT compared to the 50+ years age group (66\% [182/ $274]$ versus $55 \%$ [69/126], $p=0.025)$. The other members of the household (wife, children or other) were more in support of the SIT compared to the heads of the household (71\% [177/251] versus 50\% [74/149], $p<0.001$ ). The most reported reason for not supporting SIT by the remaining $37 \%(n=146)$ of participants was the fear that the mosquitoes would bite and cause illness. The second reported reason was that they did not understand the concept of SIT.

\section{Attitudes toward malaria}

In terms of prevention, 95\% (95\% CI: 92.9-97.3\%) of participants reported that malaria can be prevented through various preventive activities, $2 \%(n=6)$ did not know, and 3\% $(n=12)$ reported it cannot be prevented. Approximately 49\% (95\% CI: 44.2-54.2\%) reported to be using personal protective products/substances currently in their households, such as mosquito coils (55\%), burning cow dung (17\%), mosquito repellent (14\%), insecticide (7\%), burning tissues (5\%) and $2 \%$ noted use of bed nets, burning plants, closing windows, garlic and Vaseline gel. Approximately 75\% (95\% CI: 70.4-79.1\%) reported that protection against malaria was attained by indoor residual spraying (IRS). The majority (76\%) were confident that IRS kills mosquitoes and $74 \%$ were also confident that it prevents infection. At least $98 \%$ reported that it is important to allow IRS operators to spray inside their houses. Of the 400 participants, $42 \%$ reported that malaria is still a problem in the area, $53 \%$ reported that it is not a problem, and 5\% did not know.

\section{Malaria practices and treatment seeking behaviour}

Of the 400 participants, only nine (2\%) reported having bed nets in the households. We found that of these nine with bed nets, five were used by the mothers, three by the fathers, and one by the sister in the households. Only one bed net was not used as a result of wear-andtear. Approximately $99 \%$ of the houses were being 


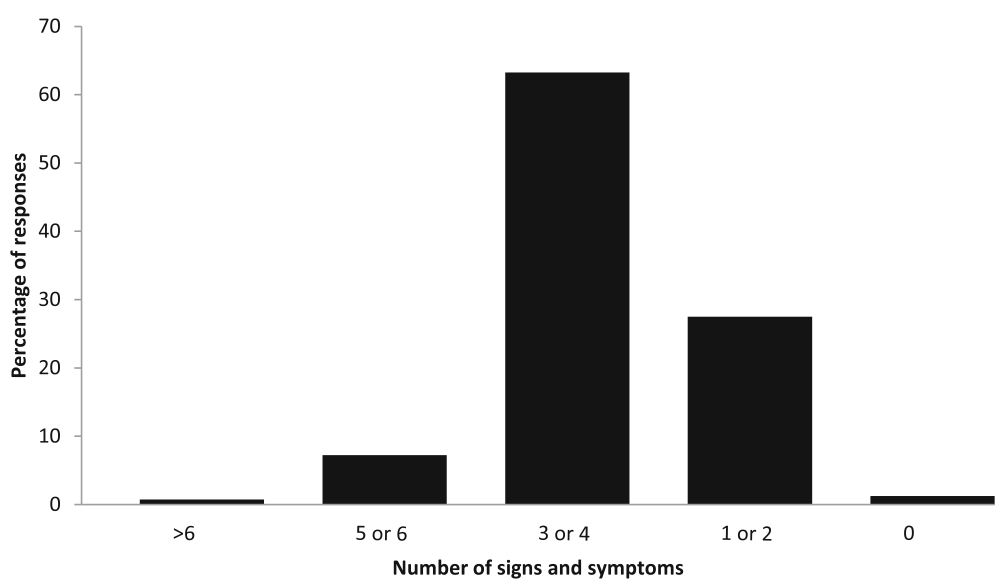

Fig. 3 Number of malaria signs and symptoms identified by the participants in Mamfene, Jozini, KwaZulu-Natal, South Africa, 2015

sprayed on an annual basis, with the other remaining $1 \%$ reporting that houses were new in the area. About two thirds $(66 \%)$ of the houses were last sprayed in 2014 . The reason given for non-spraying was mainly due to houses being locked and the homeowner not being available during the spraying schedule. The majority (99\%) reported that they would seek treatment from a health care facility if anyone in the household were to develop signs and symptoms suggestive of malaria. Approximately $63 \%$ reported treatment seeking within $24 \mathrm{~h}$ of experiencing symptoms.

\section{Discussion}

In SA, this is the first study that has been carried out to provide baseline information on malaria related knowledge, attitude and practices at community level, as the first step to introduce SIT.

Table 2 Participants knowledge of the sterile insect technique (SIT) in Mamfene Jozini, KwaZulu-Natal, South Africa 2015

\begin{tabular}{lll}
\hline Knowledge questions & & \\
\hline Participants heard about SIT before & $n=400$ & $\%$ \\
Yes & 36 & 9.0 \\
No & 364 & 91.0 \\
Where did participants hear about SIT & $n=36$ & \\
Community meetings & 30 & 83.3 \\
Health care facility & 1 & 2.8 \\
Neighbour & 3 & 8.3 \\
Church & 2 & 5.6 \\
Participants' understanding on SIT & $n=36$ & \\
To assist in elimination of malaria & 33 & 91.0 \\
Do not know & 3 & 9.9 \\
Participants' opinion on mosquito release & $n=400$ & \\
Those who are for it & 251 & 63.0 \\
Those who are against it & 149 & 37.0 \\
\hline
\end{tabular}

Adequate knowledge, encouraging attitudes, and suitable treatment seeking behaviour were demonstrated by the participants in the community of Mamfene. However, few people had heard about SIT and older respondents raised concerns about SIT as a malaria control strategy. The lack of awareness and concerns should be addressed as part of an SIT communication initiative.

The community's knowledge on malaria was generally good; however, more than half of the participants also indicated that they would like to acquire more knowledge regarding malaria. The most identified sources of information by the participants were the local health facility, radio and community meetings. Participants felt that community health promoters contributed very little information about malaria and information leaflets or booklets were not distributed adequately. We found that almost all of the participants knew about malaria and correctly associated malaria with mosquito bites, congruent with the findings of studies in Mpumalanga province [25] and neighbouring Swaziland [24]. Only about a third of the participants correctly identified the female mosquito as the carrier of infection. This will be an important point to consider when developing community health awareness messages for the SIT project, given that only non-biting male mosquitoes are released into the environment during SIT.

More than half of the participants were able to identify the most common signs and symptoms of which were headache, fever, feeling cold and vomiting. This is an important result as these are the early symptoms experienced by individuals who are infected with malaria. It also matches the WHO target for knowledge [1] and is also in accordance with findings in other studies in endemic settings [24-26].

A positive attitude regarding malaria control was demonstrated. However, approximately $22 \%$ of the participants reported the use of unproven methods of malaria 
control, such as burning cow dung, that are unlikely to provide effective protection against malaria transmission. This could have been a result of individual perceptions driven by previous experiences. Additional scope of research for improving control measures has to be investigated and encouraging the community to use available conventional methods is vital. In addition, half of the participants reported not taking any personal protective measures to guard against malaria infection. This could be due to the fact that most people are dependent on the IRS programme or information claiming that malaria is no longer a threat in their area (reported prevalence of malaria in $\mathrm{KZN}$ is $<1$ case per 1000 population) [3]. According to a study in Mpumalanga province in 2008 on knowledge and practices towards malaria, approximately a quarter of the participants reported not to use any personal protection against malaria [25].

The majority of participants reported that their households are sprayed on an annual basis and two-thirds noted that they were last sprayed in 2014. This finding is below what is expected according to the WHO guideline on IRS coverage which recommends a minimum of $80 \%$ within the targeted communities [6]. Although IRS coverage was less than expected, the majority of participants were confident that spraying kills mosquitoes and prevents infection. While bed nets have never been part of the vector control intervention in SA, the Department of Health encourages use of bed nets for personal protection. However, use of bed nets was low with only $2 \%$ of the households reported having bed nets, possibly because SA does not have a bed nets distribution programme. The last time Mamfene was supplied with bed nets was in 2000 by the Medical Research Council of South Africa (MRC) during an awareness campaign [27].

The majority of participants reported seeking treatment at a local health facility. This could be due to the perceived quality and accessibility to health care facilities in KZN. More services are now accessible and testing is also done on site in local facilities, including active case finding in the province [27]. Approximately two-thirds of the participants in the study reported that they sought treatment within $24 \mathrm{~h}$ after having experienced any signs and/or symptoms of malaria. No mention of traditional healers was reported.

More than half of the participants were in support of the SIT strategy. Participants aged 50 years and younger and other members of the household were more in support of the SIT. Participants raised concerns of being afraid of mosquitoes and fearing the unknown. These are concerns that should be addressed during awareness campaigns.

A limitation of the study was that participants' migration history was not considered. Given the degree of cross-border movement between Swaziland, Mozambique and KZN this could have an impact on the participants who reported that malaria was still a problem and not having enough information on malaria. According to Moonasar et al. 2013, it was reported that approximately $50 \%$ of infections reported in KwaZulu-Natal and approximately $80 \%$ reported in Mpumalanga are imported malaria cases mostly from Mozambique [5]. Additionally, the three sites from which participants were sampled from were conveniently selected, thereby limiting the representativeness and generalizability of the findings. However, participants were randomly selected and the study utilized a statistically calculated sample size giving sufficient power for the study.

\section{Conclusion}

We reported findings of the knowledge, attitudes and practices of the Mamfene community, an endemic malaria province in South Africa. Some key findings were that the community had reasonable knowledge of malaria transmission, but showed an interest in learning even more. Furthermore, the community were in support of passive vector control strategies such as IRS, and SIT will therefore be a supplementary passive vector control intervention. Although SIT was largely supported, a substantial proportion of the community will require more information from communication platforms identified here. This communication can be channelled through the health facility staff already involved in providing basic malaria and malaria control messages without the need for additional resources apart from additional training on SIT to ensure the message to the community is accurate and appropriate. The SIT campaign should also communicate the technology to the community to cover all age groups and to translate the scientific terminology to simple language.

\section{Additional files}

Additional file 1: KZN KAP_Questionnaire1. (XLSX 189 kb)

Additional file 2: KZN KAP Data. (DOCX $38 \mathrm{~kb}$ )

\section{Acknowledgements \\ The authors would like to acknowledge the KwaZulu-Natal Department of Health (S. Ngxongo, J. Zikhali and E. Raswiswi) and Khumbulani Hlongwana for allowing us to adapt his questionnaire in addressing our objectives and all his support in this study. We are also grateful to Dorothy L. Southern for providing scientific writing advice and critically reviewing this manuscript.}

\section{Funding}

The study was funded by the South African Field Epidemiology Training Programme at the National Institute for Communicable Diseases; International Atomic Energy Agency, the Industrial Development Corporation and the South African Nuclear Energy Corporation (Necsa) through its Nuclear Technologies in Medicine the Biosciences Initiative (NTeMBI) - a national platform funded by the Department of Science and Technology; Global Diseases Detection/CDC grant (U19GH000622-01 MAL01). 


\section{Availability of data and materials}

All data generated or analysed during this study are included in this published article (Additional files 1 and 2).

\section{Disclaimer}

The findings and conclusions in this report are those of the authors and do not necessarily represent the official position of the US Centers for Disease Control and Prevention (CDC).

\section{Authors' contributions}

PNM carried out the experiments and wrote the first and subsequent drafts of the manuscript. LK supervised the project and contributed to subsequent writing of the manuscript. AM participated in the study design, analysis and interpretation of results and reviewed the manuscript. HDM participated in the analysis. LLK conceived and supervised the project, and contributed to the subsequent interpretation and writing of the manuscript. All authors read and approved the final manuscript.

\section{Ethics approval and consent to participate}

Written consent was given by all participants. The protocol was approved by the Faculty of Health Sciences Research Ethics Committee of the University of Pretoria (Protocol Number: 217/2015) and KwaZulu-Natal Health Research and Knowledge Management (Protocol Number: HRKM 242/15).

\section{Consent for publication}

Not applicable.

\section{Competing interests}

The authors declare that they have no competing interests.

\section{Publisher's Note}

Springer Nature remains neutral with regard to jurisdictional claims in published maps and institutional affiliations.

\section{Author details}

${ }^{1}$ South African Field Epidemiology Training Programme, National Institute for Communicable Diseases, Johannesburg, South Africa. ${ }^{2}$ School of Health Systems and Public Health, Faculty of Health Sciences, University of Pretoria, Johannesburg, South Africa. ${ }^{3}$ Division of Global Health Protection, U.S. Centers for Disease Control and Prevention (CDC), Pretoria, South Africa. ${ }^{4}$ Wits Research Institute of Malaria, School of Pathology, Faculty of Health Sciences, University of the Witwatersrand, Johannesburg, South Africa. ${ }^{5}$ Centre for Opportunistic, Tropical \& Hospital Infections, National Institute for Communicable Diseases, Johannesburg, South Africa.

Received: 29 November 2016 Accepted: 11 July 2017

\section{Published online: 20 July 2017}

\section{References}

1. World Health Organization: Malaria elimination: A field manual for low and moderate endemic countries. Geneva: WHO 2007.

2. World Health Organization: World Malaria Report, 2015. Geneva: WHO 2015. [http://www.who.int/malaria/media/world-malaria-report-2015/en/] (accessed 27July 2016).

3. South African National Department of Health: National Malaria Statistics. Pretoria: NDoH 2013

4. Maharaj R, Raman J, Morris N, Moonasar D, Durrheim D, Seocharan I, Kruger P, Shandukani B, Kleinschmidt I. Epidemiology of malaria in South Africa: from control to elimination. SAMJ: South African Medical Journal. 2013; 103(10):779-83.

5. Moonasar D, Morris N, Kleinschmidt I, Maharaj R, Raman J, Mayet N, Benson F, Durrheim D, Blumberg L. What will move malaria control to elimination in South Africa? SAMJ: South African Medical Journal. 2013;103(10):801-6.

6. World Health Organization: Indoor residual spraying: use of indoor residual spraying for scaling up global malaria control and elimination: WHO position statement. 2006.

7. Coetzee M, Kruger P, Hunt R, Durrheim D, Urbach J, Hansford C. Malaria in South Africa: 110 years of learning to control the disease. SAMJ: South African Medical Journal. 2013;103(10):770-8.
8. Brooke B, Koekemoer L, Kruger P, Urbach J, Misiani E, Coetzee M. Malaria vector control in South Africa. SAMJ: South African Medical Journal. 2013; 103(10):784-8.

9. Klassen W. Introduction: development of the sterile insect technique for African malaria vectors. Malar J. 2009;8(2):1.

10. Benedict $M Q$, Robinson AS. The first releases of transgenic mosquitoes: an argument for the sterile insect technique. Trends Parasitol. 2003;19(8):349-55.

11. Dyck V, Hendrichs J, Robinson A. Sterile insect technique: principles and practice in area-wide integrated Pest management. Dordrect, Netherlands: Springer; 2005.

12. Vreysen M. Principles of area-wide integrated tsetse fly control using the sterile insect technique. Med Trop. 2001;61(4-5):397-410.

13. Dame D. Genetic control by sterilized mosquitoes. Agricultural Research Senvice (USA): Reprints-US Department of Agriculture; 1985.

14. Snow JW. Radiation, insect and eradication in North America. An overview from screwworm to bollworm. Proceedings of a Symposium Jointly Organized by IAEAVFAO: IAEA-SM-301/329: 1987.

15. Barnes BN. South Africa's fruit fly SIT programme - The Hex-River Valley pilot project and beyond. Proceedings of the 6th International Symposium on Fruit Flies of Economic Importance, Stellenbosch, South Africa, 6-10 may 2002. Isteg Scientific Publications; 2004.

16. Munhenga G, Brooke BD, Chirwa TF, Hunt RH, Coetzee M, Govender D, Koekemoer LL. Evaluating the potential of the sterile insect technique for malaria control: relative fitness and mating compatibility between laboratory colonized and a wild population of anopheles arabiensis from the Kruger National Park, South Africa. Parasit Vectors. 2011:4:208.

17. Mouatcho JC, Munhenga G, Hargreaves K, Brooke BD, Coetzee M, Koekemoer LL. Pyrethroid resistance in major African malaria vector anopheles arabiensis from Mamfene, northern KwaZulu-Natal, South Africa. S Afr J Sci. 2009;105:127-31.

18. Brooke BD, Robertson L, Kaiser ML, Raswiswi E, Munhenga G, Venter N, Wood OR, Koekemoer LL. Insecticide resistance in the malaria vector Anopheles arabiensis in Mamfene, KwaZulu-Natal. South African Journal of Science. 2015;111(11-12):1-3.

19. Bamidele J, Ntaji M, Oladele E, Bamimore O. Community participation in malaria control in olorunda local government area, osun state, southwestern Nigeria. African Journal of Infectious Diseases. 2012;6(2):24-8.

20. Alphey L, Benedict M, Bellini R, Clark GG, Dame DA, Service MW, Dobson SL. Sterile-insect methods for control of mosquito-borne diseases: an analysis. Vector-Borne and Zoonotic Diseases. 2010;10(3):295-311.

21. Statistics South Africa: Census 2012. [http://www.statssa.gov.za/publications/ SAStatistics/SAStatistics2012.pdf] (accessed July 2016).

22. Lehohla P. Census 2011 Municipal report KwaZulu-Natal. Statistics South Africa. Pretoria, South Africa. 2012. [http://www.statssa.gov.za/census/ census_2011/census_products/KZN_Municipal_Report.pdf]

23. Dayi C, Grayii A. Health and Related Indicators. Published by Health Systems Trust. South African Health Review 2008;119(3):342-55.

24. Hlongwana KW, Mabaso ML, Kunene S, Govender D, Maharaj R. Community knowledge, attitudes and practices (KAP) on malaria in Swaziland: a country earmarked for malaria elimination. Malar J. 2009;8:29-2875-8-29.

25. Hlongwana KW, Zitha A, Mabuza AM, Maharaj R. Knowledge and practices towards malaria amongst residents of Bushbuckridge, Mpumalanga, South Africa. African Journal of Primary Health Care \& Family Medicine. 2011;3(1):9.

26. Forero DA, Chaparro PE, Vallejo AF, Benavides Y, Gutiérrez JB, Arévalo-Herrera M, Herrera S. Knowledge, attitudes and practices of malaria in Colombia. Malar J. 2014;13:165.

27. National Department of Health: Malaria Elimination Strategy for South Africa 2012-2018. 2012. 